\title{
Signal Processing: Passive Acoustic in Fisheries and Marine Mammals
}

\author{
Lubis $\mathbf{Z M}^{1 *}$, Mujahid $\mathbf{M}^{2}$, Harahap $\mathbf{M S}^{3}$ and Tauhid $\mathbf{M}^{4}$
}

${ }^{1}$ Department of Marine Science and Technology, Bogor Agricultural University, Indonesia

${ }^{2}$ Bandung Institute of Technology, Bandung, Indonesia

${ }^{3}$ Department of Marine Fisheries and Livestock Production, North Labuhanbatu Goverment, Indonesia

${ }^{4}$ Department of Marine Fisheries, Bima Goverment, Indonesia

\begin{abstract}
Signal processing with passive acoustic in fisheries and marine science learn the sound frequency range of fish, the intensity of the sound amplitude, sound fluctuations, and shape the sound patterns of the fish. Application of passive acoustic filed for marine fisheries and science detects the sound frequency range of fish, the intensity of the sound amplitude, sound fluctuations, and shape the sound patterns of the fish. Passive acoustic methods used to monitor marine mammals expressed. In general, the signal obtained from the ranimal record sounds is poor and difficult to determine from which directions it is produced, therefore it requires that require amplification/ strengthening. Bioacoustic research is needed to identify the communication language (acoustic communication) in mammals. Bioacoustic detect mammal-produced frequency ranges of sound, amplitude intensity of sound, voice fluctuation, and form sound patterns of mammals. Studying bioacoustic is inseparable from the science of underwater acoustics, biology of mammals, and the study of mammalian behavior. Generally bioacoustic include physiology of mammals organ that produce sound, earnings voice mechanism, sound characteristics of mammals, mammals sound-approaching mechanism, the hearing capacity of fish, and the evolution of the auditory system, and to obtain the frequency range of each sound produced by the dolphins (mammals). Environmental conditions and parameters (salinity and temperature) will greatly affect the value of the intensity and frequency generated from the target, the more extreme the environmental conditions, the lower value of the intensity and frequency generated.
\end{abstract}

Keywords: Signal Processing; Passive acoustic; Bioacoustic; Mammals; Frequency

\section{Introduction}

Acoustics is the science which deals with sound waves and wave propagation in a medium. Thus, marine acoustics is the study of sound waves and penjalarannya (propagation) in the medium of seawater (occurring in the water column). Marine acoustics is a marine field to detect targets in the water column and base peairan using sound waves. With the application of marine acoustics will facilitate researchers to determine the object that is in the water column and seabed in the form of plankton, fish, content of the substrate and the ship foundered [1].

Acoustic method used to obtain estimation of fish can use basic methods such echocounting and echo integration. Echo counting can compute density fish when the sampled volume is low, value echo of a single fish can be easily separated and computing one by one. Echo counting method used in rarely suspect the abundance of fish are clustered. It caused the density of fish is not homogeneous and high in general, so will cause the overlap of the echo fish. Echo of the fish that were in seabed has a stronger signal than with fish located in seabed [1].

Passive acoustic methods used to monitor marine mammal belonging to [2]. In general, the signal obtained from the sound recording valuable animals are so weak that require amplification/ strengthening and difficult to determine where it came from the direction of sound. The basic concept of passive acoustics in mammals is to detect sounds when the mammals are in the area of measurement. Measurement is done by using the software, and also to listen. Passive acoustic methods are also used by the military in developing the security system of the attacker under water in the estuary area by recording the sound generated from underwater divers [3]. Passive acoustics are not separated by their sound (Sound). Sound is a mechanical wave of energy that is changing the pressure in the medium (air or water) when the wave moves. These pressure changes detected by our hearing and transmitted to the brain for interpretation. Diinterpratasikan sound waves by the wavelength (wavelength), amplitude, frequency and intensity (in decibels-dB), which can be seen in Figure 1 [4].

The applications of passive acoustics are usually referred to as passive sonar where the application of this science is usually called the ilu bioacoustics (Bioacoustic). Bioacoustics (Bioacoustics) is a discipline that combines biology and acoustics usually refers to a study on sound production, dispersion through elastic media, and reception in animals, including humans. This involves neurophysiology and anatomy for the production and detection of sound, and the relationship with the acoustic signal dispersion medium. The findings in this area provide evidence for us about the evolution of the acoustic mechanism, and from there, the evolution of animals used [1]. Passive sonar system can be seen in figure 2 on the mechanism of passive sonar equation [5] (Figure 2).

Source level (SL) is the amount of noise emitted by a transducer. Transmission Loss (TL) is the intensity of the sound energy is reduced when propagating in the medium. DT (Detection Threshold) is the signal-to-noise ratio necessary and the target signal is a function of the receiver. Acoustic science is highly developed in dolphins; previous researchers have emphasized the recording and analysis of vocalization [6-10]. Research of bioacoustics is needed to be able to know language of communication (Acoustic communication) in mammals. Bioacoustics

${ }^{*}$ Corresponding author: Muhammad Zainuddin Lubis, Department of Marine Science and Technology, Bogor Agricultural University, Kampus IPB Dramaga Bogor, Jl. Raya Darmaga, Bogor, Jawa Barat, 16680 Indonesia, Tel: 622518628448; E-mail: lubiszainuddin@gmail.com

Received May 05, 2016; Accepted May 28, 2016; Published June 08, 2016

Citation: Lubis ZM, Mujahid M, Harahap MS, Tauhid M (2016) Signal Processing Passive Acoustic in Fisheries and Marine Mammals. J Biosens Bioelectron 7: 208. doi:10.4172/2155-6210.1000208

Copyright: ( 2016 Lubis ZM, et al. This is an open-access article distributed under the terms of the Creative Commons Attribution License, which permits unrestricted use, distribution, and reproduction in any medium, provided the original author and source are credited. 


\section{A Sound Wave:}

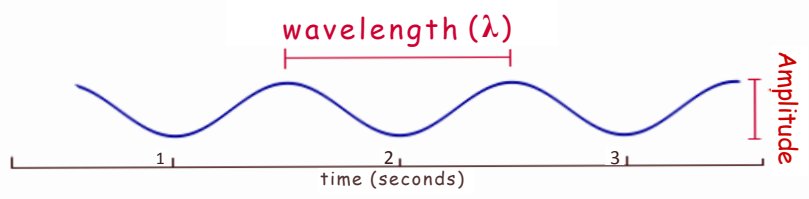

period = time of one cycle (wavelength)
above: one second from crest ti crest

frequency $=$ number of cycles/second

above: $1 \mathrm{cycle} / \mathrm{sec}=1 \mathrm{Hertz}(\mathrm{Hz})$

Figure 1: Sound waves.

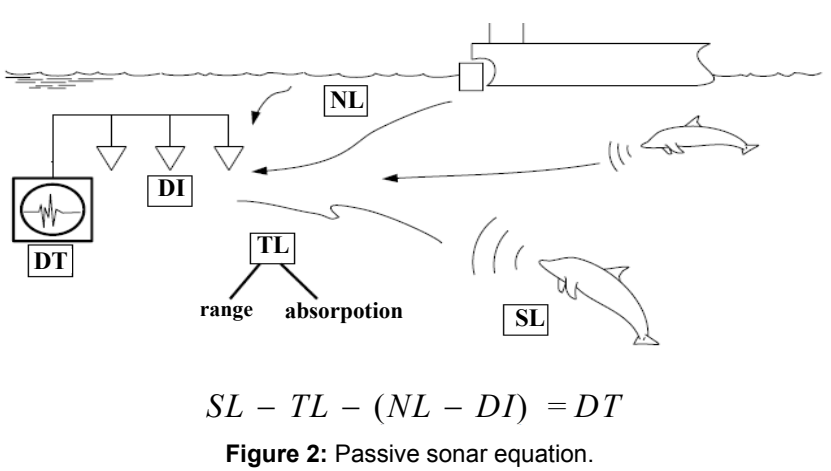

not be separated from the use hydrophones as lat voice recorder in which the acoustic pressure recorded on hydrophone is a source of pressure on the sea disturbance time $(\Delta \mathrm{P})$ relative to the pressure of the ocean in the background on the recording medium depth water.

Science bioacoustics also learn about stridulatory, stridulatory Voice is the sound produced by moving or menggemertakkan body parts, for example: fins, teeth and other body parts were hard [10]. Bony fishes (teleost) has a sound produced from flapping fin and some other types of sound stridulatory has a large amplitude, which are spread uniformly throughout the frequency. Frequency achieved can range up to more than $6000 \mathrm{~Hz}$ [11]. Environmental conditions and parameters (salinity and temperature) will greatly affect the value of the intensity and frequency generated from the target, the more extreme the environment will certainly leave a low value of the intensity and frequency generated [4,12-14]. Bioacoustics application of science in the fishery usually applied using marine mammals for example the usual whales dsebut with echolocation in whales. Echolocation is how whales use sound to locate objects (e.g. prey) and determine their position in the wide ocean in three dimensions. Reflected sound time after hitting the target, then the echo occurs. Whales emit short sound called clicks and can determine the location of the object through the echo is formed. The distance of the object can be determined by calculating the length of echo back to them (the whale). Schematically echolocation of whales can be seen in Figure 3.

Figure 3 above shows the time of 6 seconds between clicking sound that has come out with the echo back. Half the time required for a click to achieve the object, meaning that the object taken within 3 seconds. The speed of sound in water is $1500 \mathrm{~m} / \mathrm{s}$, then the object is located at a distance of 4500 meters from the whales away ( 3 seconds 1500 metres times/second $=4500 \mathrm{M})$.

Echolocation is indicate that the whale has a very good sound production and sound reception system. Reception system sound on cetaceans is already very advanced, because of the direction and timing of the return echo, these animals can determine the shape of objects and material. Cetaceans can determine the degree of sound like a man, even to the tenth of milliseconds, a higher value than human resources.

\section{Method}

The entire acoustic sensing using microphones and transducers to detect acoustic energy and then convert it into an electrical signal [15]. For recording underwater sounds using a hydrophone (Figure 4). Hydrophone is an underwater microphone that captures the acoustic signals then converts this energy into electrical energy and is used in passive acoustic systems. Measurement of the sound signal that we want to know is to measure the Signal to Noise Ratio (SNR) is the ratio between the votes received signal level (received level of a sound signal) to the background noise level (background noise level) [16] (Figure 4).

Bioacoustics using passive instrument commonly called hydrophones is an instrument that serves to listen to underwater sounds. This tool converts the sound coming from the water being electrically signal, and then can be amplified, analyzed, or played in the air [15]. Hydrophones usually a piezo-electric ceramic plate [15]. Dolphin EAR hydrophones capable of detecting the frequency of the sound at 1-2 Hz. The lowest threshold for human hearing only able to listen to the sound frequency of $18-20 \mathrm{~Hz}$ [4]. The voices outside the normal threshold of human hearing can be heard using the Dolphin EAR hydrophones are equipped with Raven lite 1.0 software. Image recording a set of tools in bioacoustics hydrophones can be seen in Figure 5 [17].
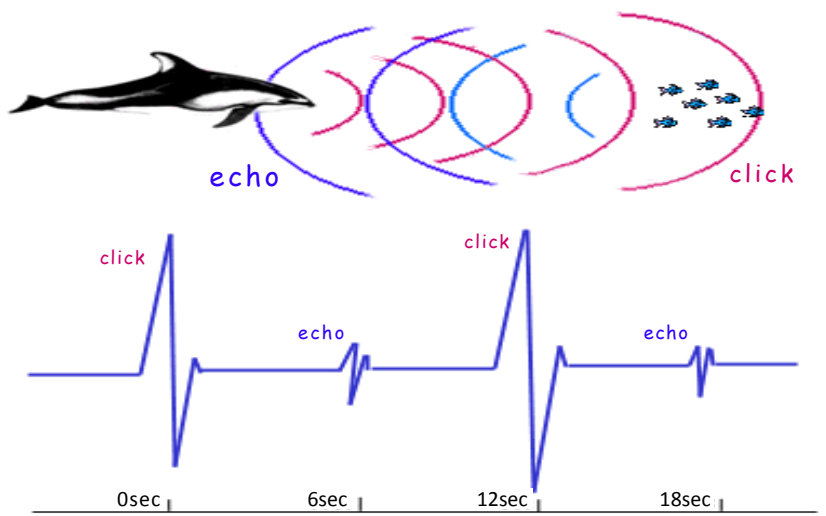

Figure 3: Whale echolocation in the application of bioacoustics.

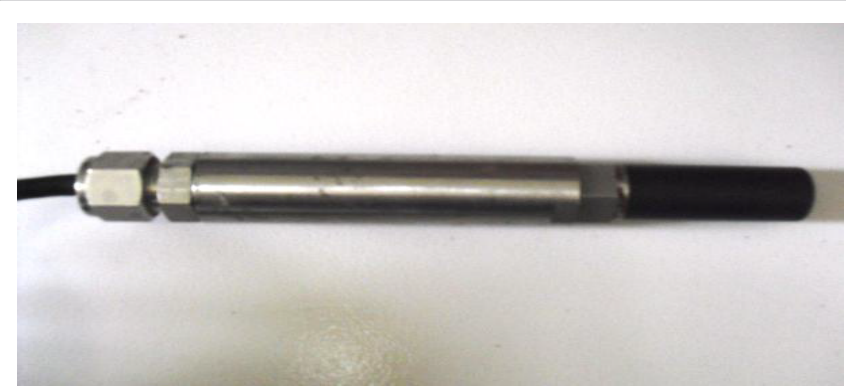

Figure 4: Hydrophone type SQ3. 


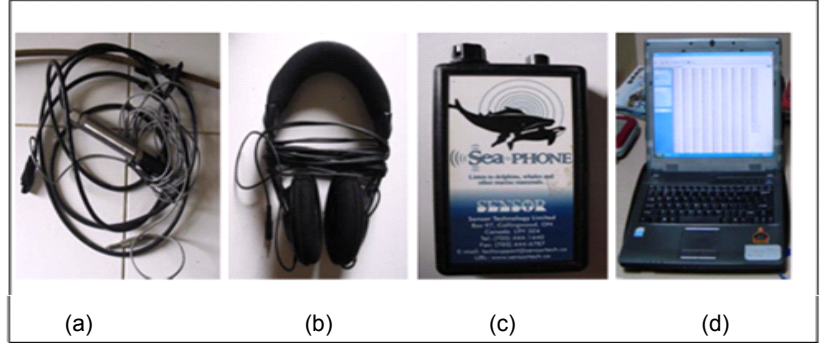

Figure 5: Set voice recorders, (a) Hydrophone, (b) Headphone, (c) the power supply/battery, and (d) laptop for data logging and data processing [17].

\section{Data processing of sound}

The voice data that has been recorded by a digital voice recorder in the form of extensions *. VY4, re-recorded using the Advanced Sound Recorder 6.0 program that will generate the voice data in the form of extension *.mp3. Furthermore, the voice data is already in the form of extension ${ }^{*}$.mp3 is stored in the form of extensions ${ }^{*}$. WAV using Wavelab 6.0 program. The process of data analysis can be seen in Figure 5.

After the voice data is in the form of extensions ${ }^{*}$. WAV, sound process is then performed to eliminate interference (noise) using Cool Edit Pro 2.0 program. The voice data has been cleared of interference (noise) and then processed using software Wavelab 6.0. The data transformation from form to form sound figures using FFT data analysis in program Wavelab 6.0a is then performed handoff from the form of the extension *.WAV be ${ }^{*}$.txt.

After becoming a form of ${ }^{\star}$.txt, data processed using Microsoft Excel program simply averages the numbers per $1000 \mathrm{~Hz}$ and obtained data that has a range of numbers between $0-22000 \mathrm{~Hz}$. The average is then converted into the form of decibels using the equation:

\section{Fourier transformation in acoustic}

The frequencies are $w_{n}=\frac{n \pi}{T}$ and $\Delta w=\frac{\pi}{T}$

Therefore (1) and (2) are represented as

$$
\tilde{f}(x)=\frac{1}{2 \pi} \sum_{n=-\infty}^{\infty}\left(e^{i w x} \int_{-T}^{T} e^{-i w t} f(t) d t\right) \Delta w
$$

Since, on one hand the function with period $T$ has also the period's $k T$ for any integer $k$, and on the other hand any non-periodic function can be considered as a function with infinite period, we can run the $T$ to infinity, and obtain the Riemann sum with $\Delta w \rightarrow \infty$, converging to the integral:

$$
\tilde{f}(x)=\frac{1}{2 \pi} \int_{-\infty}^{\infty}\left(e^{i w x} \int_{-T}^{T} e^{-i w t} f(t) d t\right) d w
$$

The integral (7) suggests the formal definition: The function $F(w)$ is called a Fourier Transform of function $f(x)$ if:

$$
F(w):=F\{f(t)\}:=\int_{-\infty}^{\infty} e^{-i w t} f(t) d t
$$

The function is:

$$
F^{-1}\{F(w)\}:=\frac{1}{2 \pi} \int_{-\infty}^{\infty} e^{i w x} F(w) d w
$$

\section{Fourier transform of a time shifted signal}

We'll show that a Fourier transform of a signal which has a simple time shift is:

$$
F\left\{x\left(t-t_{0}\right)\right\}=e^{-j \omega t_{0}} X(j \omega)
$$

Example: Linearity and Time Shift is Consider the signal (linear sum of two time shifted rectangular pulses)

$$
x(t)=0.5 x_{1}(t-2.5)+x_{2}(t-2.5)
$$

where $x_{1}(t)$ is of width $1, \mathrm{x}_{2}(t)$ is of width 3 , centred on zero (Figure 6)

Using the FT of a rectangular pulse L10S7

$$
X_{1}(j \omega)=2 \sin (\omega / 2) / \omega, X_{2}(j \omega)=2 \sin (3 \omega / 2) / \omega
$$

Then using the linearity and time shift Fourier transforms properties

$$
X(j \omega)=e^{-j 5 \omega / 2}((\sin (\omega / 2)+2 \sin (3 \omega / 2)) / \omega)
$$

\section{Result and Discussion}

Then from the data obtained can be displayed in the form of a graph desired, i.e., chart bar chart and graph diagram stock for the left and right sound data and image examples spectrum of the sound generated from Raven lite 1.0 software can be seen in Figure 7 and spectrograms results voice recording derived from the spectrum of dolphin and processed using GIS View 2.7.1 can be seen in Figure 7.

Pictured above is an image of the sound spectrum analysis with a length of recording time is $700 \mathrm{~ms}$ and the $\mathrm{y}$-axis is the magnitude of the resulting frequency spectrum of the data processed (Figure 7).

Spectral data analysis process and the characteristics of an object or target in the application of science bioacoustic is usually not separated from the aspect of transformation forier and power spectral density are used to examine the relationship between the intensity and frequency signals $[18,19]$. Density estimate 1D wavelet whistle of dolphin can be seen in Figure 8.

Figure 8 above shows the results of density estimation signal has a decomposition different, but the premises the shoulder (top of the chart) are shown in the picture to the right with a circled circle is black, it can be concluded that the center of the true voice signal is entered is
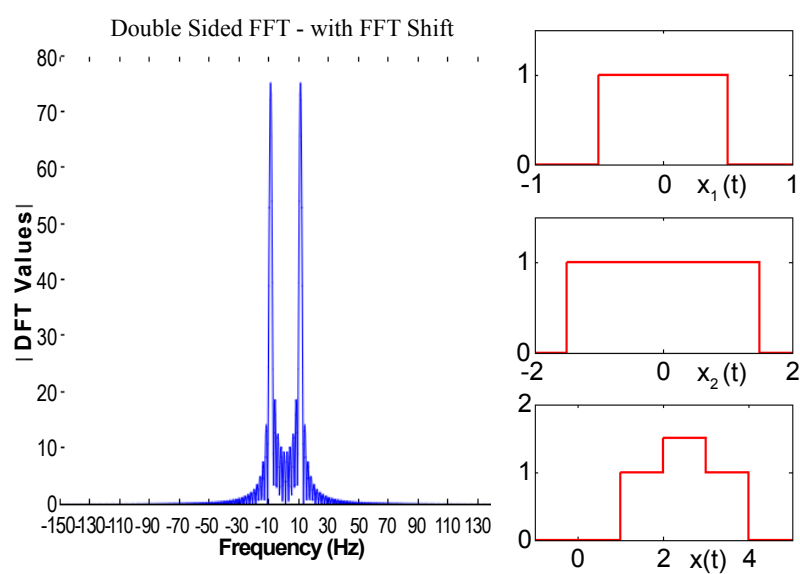

Figure 6: Linearity and time shift and FFT with FFT Shift Use MATLAB. 

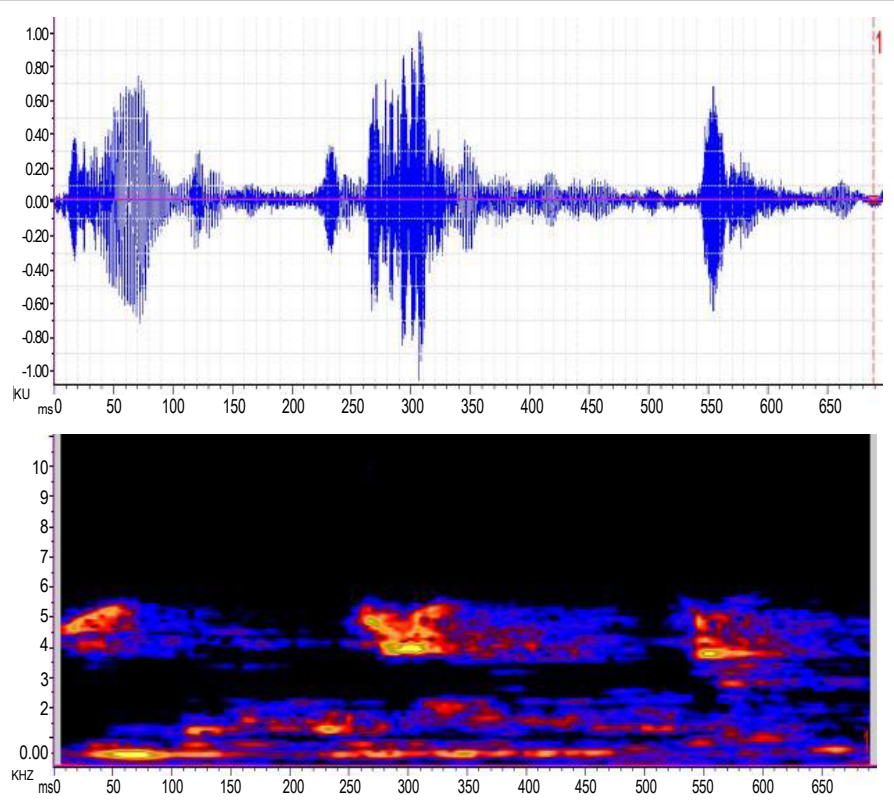

Figure 7: Pictured above is an image of the sound spectrum analysis (Figure 7) with a length of recording time is $700 \mathrm{~ms}$ and the $y$-axis is the magnitude of the resulting frequency spectrum of the data processed.
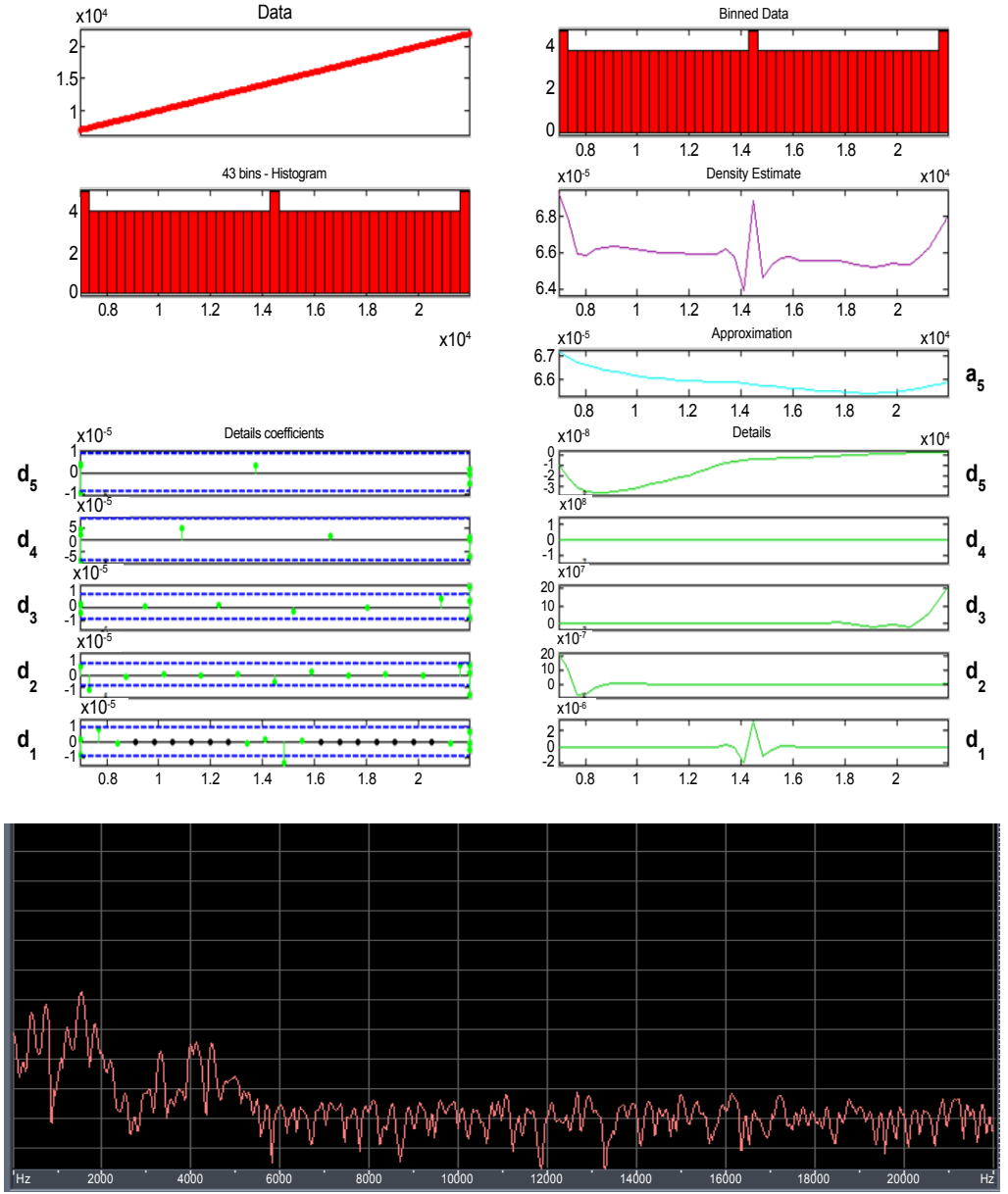

Figure 8: Fast Fourier Transform dolphin sounds.
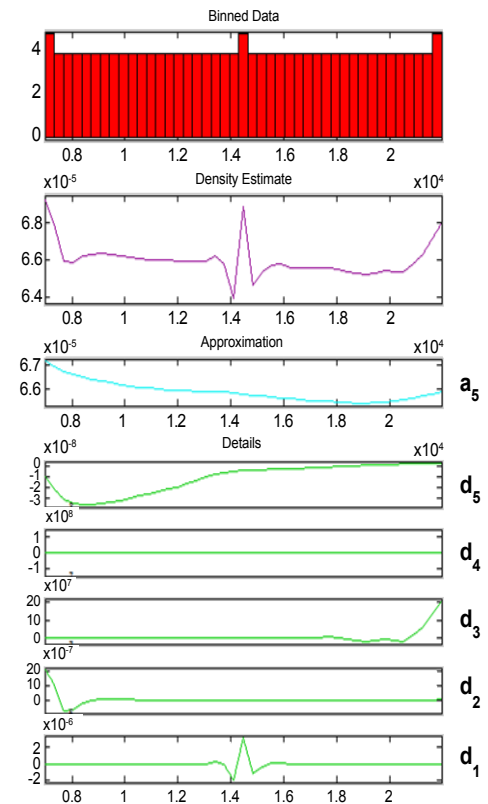
Citation: Lubis ZM, Mujahid M, Harahap MS, Tauhid M (2016) Signal Processing: Passive Acoustic in Fisheries and Marine Mammals. J Biosens Bioelectron 7: 208. doi:10.4172/2155-6210.1000208

Page 5 of 5

in the range $14 \mathrm{KHz}-15 \mathrm{KHz}$. Range of voice signal be same according in [20] (Figure 8).

\section{Conclusion}

The results of this paper show that the study of passive acoustics applied to the science of bioacoustics frequently to mammals and biota/other animals can be as a reference or references were highly accurate, high accuracy, is not harmful and destructive biota/animals to be used as a target, because acoustic method used to record only and does not send waves/sound (recording propagation of sound produced from a targets).

\section{References}

1. Simmonds J, MacLennan D (2005) Fisheries Acoustics: Theory and Practice, (2nd Edn.), Blackwell.

2. Nedwell JR, Parvin SJ (2007) Improvements to Passive Acoustic Monitoring systems. Subacoustech Ltd, London.

3. Borowski B, Alexander S, Heui-Seol R, Bunin B (2008) Passive Acoustic Threat Detection in Estuarine Environments. Stevens Institute of Technology: Maritime Security Laboratory. Proc of Society of Photographic Instrumentation Engineers 6945: 1-11.

4. Wulandari PD, Pujiyati S, Hestirianoto T, Lubis MZ (2016) Bioacoustic Characteristic Click Sound and Behaviour of Male Dolphins Bottle Nose (Tursiops aduncus). J Fisheries Livest Prod 4: 1-5.

5. Ainslie MA (2010) Principles of Sonar Performance Modelling. Springer p. 800.

6. Evans WE (1966) Vocalizations Among Marine Mammals. Marine Bioacoustics 2: $159-185$.

7. Wartzok D, Ketten DR (1999) Marine Mammal Sensory Systems. In J. E. Reynolds II, S. A. Rommel (Eds.), Biology of Marine Mammals.Washington, DC: Smithsonian Institution Press pp: 117-175.

8. Herman LM, Tavolga WN (1980) Communication Systems of Cetaceans. In L. M. Herman (Eds.), Cetacean Behavior: Mechanisms and Function. John Wiley \& Sons: New York pp: 149-197.

9. Popper AN (1980) Sound Emission and Detection by Delphinids. In L. M.
Herman (Eds.), Cetacean Behavior: Mechanisms and Functions. John Wiley \& Sons: New York pp: 1-52.

10. Pitcher TJ (1993) Behaviour of Teleost Fishes ( $2^{\text {nd }}$ Edn.). Clays Ltd. St Ives Plc. England.

11. Cook MLH, Sayigh LS, Blum JE, Wells RS (2004) Signature Whistle Production in Undisturbed Free-Ranging Bottlenose Dolphins (Tursiops truncatus). Proceedings of the Royal Society 271: 1043-1049.

12. Muhammad Z, Pujiyati S (2015) Influence of Addition of Salt Levels Against Study of Bio-Acoustic Sound Stridulatory Movement Fish Guppy (Poecilia reticulata).

13. Lubis MZ, Wulandari PD, Pujiyati S (2015) Pengaruh Penambahan Kadar Garam Terhadap Studi Bioakustik Suara Stridulatory Gerak Ikan Guppy (Poecilia Reticulata). The 1st International Conference on Maritime Development Proceeding. Tanjungpinang pp: 1-7.

14. Lubis MZ, Pujiyati Sri, Totok H, Wulandari PD (2016) Bioacoustic Characteristics of Whistle Sounds and behaviour of male Indo-Pacific bottlenose dolphins (Tursiops aduncus) in Indonesia. International Journal of Scientific and Research Publications 12: 7.

15. Simmonds MD (1992) Gradistat: A Grain SizeDistribution and Statistics Package for the Analysis of Unconsolidated Sediments. Royal Holloway University of London.

16. Good MD, Gilkey RH (1996) Sound Localization in Noise: the Effect of Signalto-Noise Ratio. The Journal of the Acoustical Society of America 99: 1108-1117.

17. Lubis MZ (2016) Identifikasi Karakteristik Whistle dan Tingkah Laku LumbaLumba (Tursiops aduncus) di Taman Safari Indonesia. Cisarua Bogor.

18. Brook D, Wynne RJ (1991) Signal Processing: Principles and Applications Edward Arnold, a division of Hodder and Stoughton Limited, Mill Road, Dunton Green. Great Britain.

19. KraussTP, Shure L, Little JN (1995) Signal Processing Toolbox: For Use with Matlab. The Mathworks Inc.

20. Moron JR, Andriolo A (2015) Preliminary Evidence for Signature and Copied Whistles among Spinner Dolphins in the Southwest Atlantic Ocean: Beacon Purpose? The Journal of the Acoustical Society of America 138: 1904-1904. 